International Journal of Engineering \& Technology, 7 (2.21) (2018) 62-65
International Journal of Engineering \& Technology
SPC
Website: www.sciencepubco.com/index.php/IJET
Research paper

\title{
A Cardio pulmonary resuscitation device for stretchers
}

\author{
Kapil Sadani ${ }^{1}$, D.A.P. Prabhakar ${ }^{2}$, Pooja Nag ${ }^{1 *}$, \\ ${ }^{1}$ Dept. of Instrumentation and Control, Manipal Institute of Technology, Manipal \\ ${ }^{2}$ Department of Mechatronics Engineering, Manipal Institute of Technology, Manipal University, Manipal \\ ${ }^{3}$ Department of Mechatronics Engineering, Manipal Institute of Technology, Manipal University, Manipal \\ *Corresponding author E-mail: pooja.ng08920@gmail.com
}

\begin{abstract}
Cardiopulmonary Resuscitation (CPR) machine is a class III medical device for use in cardiac arrests. This budget device has been fabricated in house and calibrated using data from sensors used when an expert administers CPR on a patient in the face of emergency. Also, the device has its own feedback sensorics to optimize CPR delivery primarily the full chest recoil. The device was tested on mannequin and its performance was identical to trained CPR experts in terms of rate and pressure. On fulfilling all ethical requirements, this device has the potential to be a life saver in India and worldwide.
\end{abstract}

Keywords: Cardiopulmonary resuscitation (CPR), electro-pneumatics, emergency care, feedback control.

\section{Introduction}

Cardiovascular disease (CVD) is globally considered as the leading cause of death with $80 \%$ of CVD related deaths being reported from low and middle-income countries like India [1]. Unattended cardiac arrests are fatal as the heart fails to supply oxygen to organs especially the brain where cells begin to die within a minute from the commencement of an arrest. Manual Cardiac Pulmonary Resuscitation (CPR) is accepted worldwide as a lifesaving technique in cardiac emergencies where forced circulation of oxygenated blood is ensured externally till the patient reaches the Intensive Care Unit (ICU).

The American Heart Association (AHA) is a globally accepted body that puts up goldens standards in cardiac care and CPR through certification courses. It continually provides improved guidelines through user feedback for CPR administration. As per standard AHA guidelines [2], the chest is nominally thumped 100120 times per minute to an average chest depth of 4-6 cm; rescue breaths at the rate of one per ten strokes need to be administered as well. As per a recent update, CPR needs to be administered with minimum interruptions, rescue breaths being administered parallelly ensuring full chest recoil.

CPR is a very exhausting activity and requires tremendous fitness as well as training. It has been reported that continuous CPR administration till a patient reaches the hospital could increase the chances of survival by $6 \%$ [3]. The very concept of a machine administering CPR is questionable by anesthetists and cardiologists worldwide because of the debatable efficiency and risks involved such as considerations of chest compliance, ensuring full chest recoil, rib breakage, rescue breaths etc. However, considering the patient load and the cumbersome nature of delivery, there is a dire need for developing an automated system for intelligently delivering CPR. Companies like Lucas [4] have their devices cleared for limited field trials but their efficiency is largely debated [5,6]. Moreover, affordability is a major issue considering the need in Indian scenarios.

The ethical issues concerning the administering of CPR is another factor that plays a vital role. As resuscitation practice evolves, ethical issues should also evolve. The decision of whether to start or when to terminate varies according to the victim, providers, and location. Awareness about the process should be made using appropriate language. [2]

This work reports of a prototype bed-top device for CPR administration on stretchers in ambulances and in hospitals. This is an indigenous development of an intelligent CPR device that is easy to setup and which could be operated with minimal training. This life saving device will be able to work in hospitals, ambulances, and other public places. The current invention incorporates the use of feedback sensors and training from expert (AHA certified) CPR caregivers to administer optimal CPR as per AHA (American Heart Association) guidelines. The device is the first of its kind in India, with a potential of mimicking AHA certified caregivers in CPR administration accounting for intelligent adaptation to the physique of the patient, primarily the chest compliance to attain an optimum number of uninterrupted compressions.

\section{Mechanical framework design}

With the objective of developing a robust and cost-effective hardware, a framework design was conceived in metal and developed in AUTOCAD and simulated in SOLIDWORKS. The overall framework is depicted in Fig. 1, shows CAD model of the mechanical structure with all dimensions.

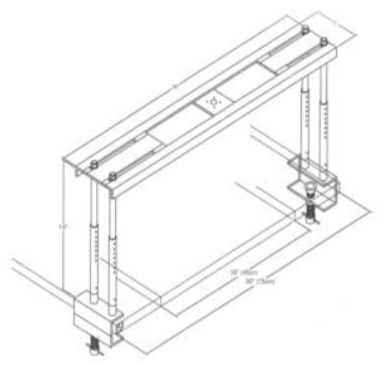

Fig.1: CAD Model of the mechanical structure with its various components and thorough dimensions. 


\section{Components}

Mild Steel (MS) sheets were used to develop guide railings for the actuator and clamps. Concentric slotted aluminum tubes were used to provide support and adjust for height variability based on patient physique. MI rods were used to design custom screws to clamp on to all standard stretcher bed sizes. All the hardware was locally procured and molded in university workshop.

The upper platform was made with two MI angles and a MI flat welded together to make guide railings for the actuator. C-Clamps were designed with MI U and slotted with custom bolt for clamping the device rigidly to any stretcher or bed. The device accounts for all variability of bed sizes both in terms of width and clamp area. Four supporting tubes have been used to support for the upper platform. The framework consists of three parts: i. Clamp ii. Supporting Rods iii. Top of the frame.

\section{Clamps}

C - Clamp has been used for clamping the device to beds or stretchers. Clamp of dimensions $(2 " \times 5$ " $\times 3$ ") have been found optimal designed using AUTOCAD (Fig. 2). The limits of clamp adjustment are made variable from standard stretcher thickness of 0.75 inches to ICU (Intensive Care Unit) beds of 2.5 inches using custom nut-bolt arrangement. The two clamps would support the entire device upright above the patient rigidly. Standard university workshop facility was used for fabricating and finishing the clamps. The clamps were tested for fatigue using SOLIDWORKS.

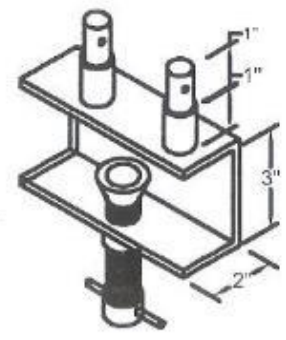

Fig. 2: CAD diagram of the clamp. The clamps are placed at the bottom of the device for setting the device to the bed.

\section{Supporting rods}

Supporting concentric slotted aluminum tubes have been used as supporting structures to support the upper platform. Since aluminum cannot be welded on mild steel as steel and aluminum melt at different temperatures, these hollow rods have been fixed on the clamp using two galvanized iron (GI), solid cylinders on top of each clamp. The solid cylinders are shaped such that the diameter of this cylinder is barely less than the inner diameter of the aluminum hollow rod so that there is a perfect fit between supporting rods and cylinders. Two GI cylinders are hence welded on top of each clamp, on which the aluminum rods are mounted and fastened using screws. Each GI cylinder has two concentric aluminum rods fixed to it to adjust the height of the frame as shown in Fig. 3. The minimum adjustable height of the supporting rods is 6" while the maximum adjustable height is 13 ". To fix the supporting rods on the top frame, four nuts have been placed and fixed rigidly.
Fig. 3: CAD model of the supporting rod to vary the height of the device, provision of holes has been done.

\section{Top of the frame}

The top frame for holding the actuator has been constructed using two MI angles welded through an I shaped mild steel slab at two ends. At the center, a square piece of mild steel has been welded to fix the actuator. The top frame has been constructed taking into consideration the reaction forces that the actuator will be bearing during the operation of the device. To hold the actuator, a center square of $3 \times 3$-inch dimension was made. To fix the supporting rods to this part of the frame, four nuts along with washers are used for a firm and secure grip. The frame is hence designed for height between 6" to $13 "$ and width between 18" to 30". The supporting rods can be moved sideways to adjust it according to the width of the hospital bed. The CAD design of the frame depicted in figure 4 shows the detailed dimensions of the frame. Fatigue analysis of the frame was done using SOLIDWORKS.

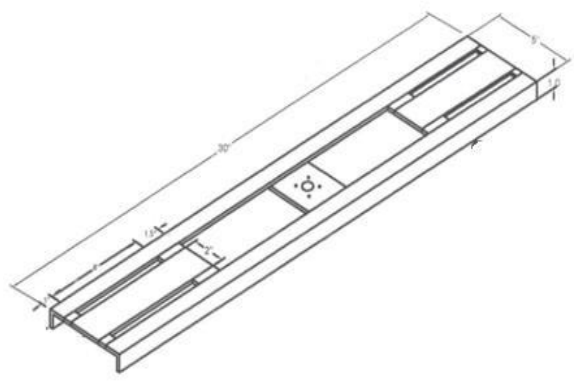

Fig 4: CAD diagram of the top of the frame.

\section{Electro-pneumatic assembly}

Double acting pneumatic cylinder (FESTO 156539) with a stroke length of fifteen centimeters was used for actuation. A 5/2 solenoid operated direction control valve (FESTO 167076) was used as the final control element. Commercial portable pneumatic compressor was used to supply necessary air with air pressure being regulated by a proportional control valve. $24 \mathrm{~V}, 3 \mathrm{~A}$ adapter was used for powering the pneumatic assembly which can be replaced by a commercial battery as well. The $5 / 2$ valve was controlled using a commercial Raspberry Pi (RBP) development board. A custom-made diaphragm was used on the piston of the double acting pneumatic cylinder.

\section{Sensor assembly}

A sensor mat was fabricated comprising button type load cells for compression application during administration of CPR and gyroscope to provide for force and acceleration feedback as depicted in Fig.5.

The modules were wirelessly connected to the (Raspberry Pi) RBP using a high tolerance Bluetooth module. A commercial AAA battery powered the sensor mat was used. The purpose of the sensor mat was to provide constant feedback to ensure full chest recoil (to a tolerance of $15 \%$ ). This mat was required to be strapped on to the patient's chest exactly below the diaphragm while installation. 

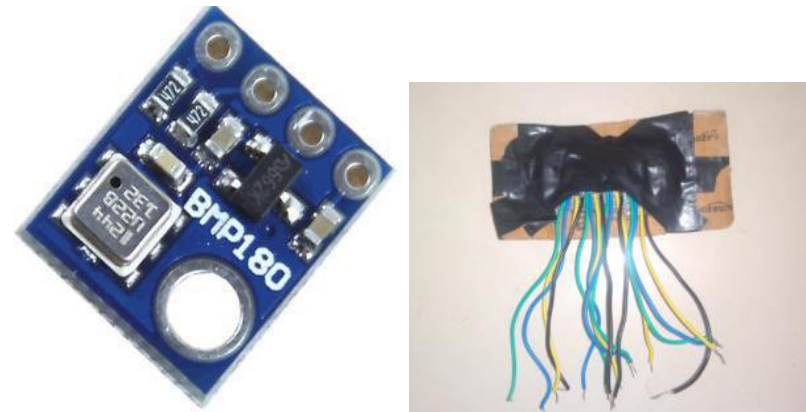

Fig.5: A sensor mat was developed and CPR professionals were requested to administer CPR on a mannequin.

\section{Experimental setup}

In the initial design phase, this sensor mat was placed on a mannequin and AHA certified CPR experts from Kasturba Medical Hospital, Manipal were asked to administer CPR on it. On application of CPR manually by professionals, the rate of compression has been observed to be 45 compressions $/ \mathrm{min}$. Nominal values of force and acceleration were determined using this data as depicted in Fig.6.

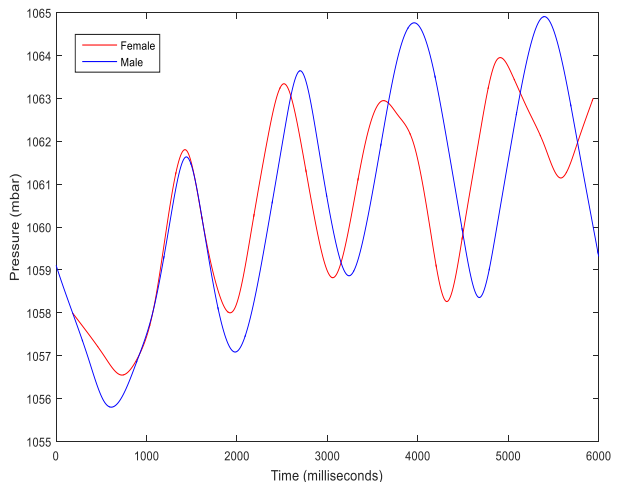

Fig.6: The graph shows Time vs Pressure plot of manual CPR for three consecutive trials. Rate: 45 compressions/min

The experiment was conducted at the simulation centre of Kasturba Medical Hospital, Manipal. The prototype was tested on mannequin with an air pressure of 5 bar pressure.

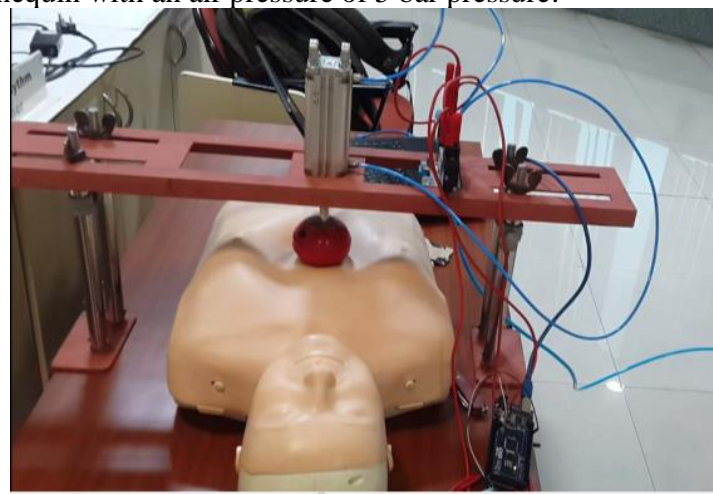

Fig.7: Testing of prototype on a mannequin at simulation centre. Foot pedal was used to generate the necessary air pressure.

A graph was plotted for the pressure exerted taking time in seconds on $\mathrm{X}$-axis and Sensor Pressure in millibar on $\mathrm{Y}$ - axis. Rate of compressions has been found to be 60 compressions/min in all the trials.

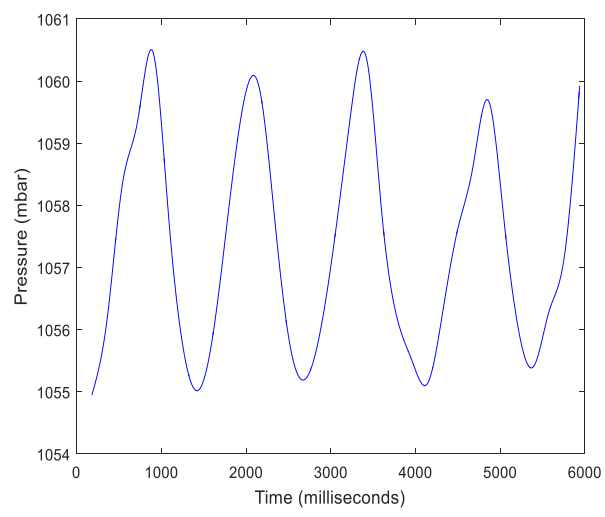

Fig.8: The graph shows the Time vs Pressure plot of CPR administered by the prototype. Source Pressure $=3$ bar (X-axis: time (sec), Y-axis Pressure(mbar))

Fig. 6 and Fig. 8 shows the variation of pressure with time for manually administered and prototype administered CPR.We propose of two control mechanisms, one in which feedback sensorics are used to control stroke depth, acceleration, and force as in Fig.9.

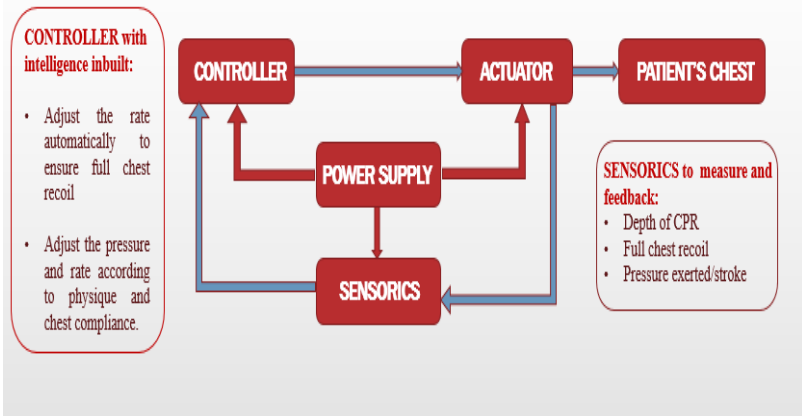

Fig.9: Flowchart of the control system with feedback control

The second control mechanism is feed forward mechanism trained with data from expert CPR administration. The second is currently in progress under development.

\section{Results and discussion}

All the components were simulated for stress and fatigue. Mean time between failures was estimated to be 522 days. The device was tested on a mannequin and the pressure vs time graph is depicted in Fig.6 and Fig.8.

The current, manual process of chest compression cannot be sustained for a long time, especially in ambulance and other settings and often trained personnel are in short supply or not available on the spot when required. A portable, automated system can be made available in such locations. It would be capable of functioning in any setting without any time constraints.

There is minimal recurring cost for such a system as opposed to monthly salary expense for a trained person. In this aspect, an automated system is far superior. However, the physique of the patient and the constraints limit efficacy of the machine such as those in an ICU, ambulance or for that matter any other public place. Hence the free use of such devices bypassing medical expert supervision is not recommended.

Our attempt is to bridge the gap considering both machine feedback (using sensors) and human feedback (by training our device) to administer effective CPR on any patient.

\section{Conclusion}

The device proposed hereof is the first prototype and needs to be optimized further with more training data and pass all ethical clearances. However, the team has been able to put up a CPR device at one tenth the cost of the FDA cleared manufacturer from 
Europe. The device performance was equivalent to that of experts from Kasturba Medical College, Manipal University.

\section{Acknowledgements}

We would like to thank Professor Nagaraj, Mechanical and Manufacturing Engineering Department, Manipal Institute of Technology, Manipal and Mr. Yajannath, Workshop Superindent, MIT Workshops, Manipal Institute of Technology, Manipal for their suggestions and inputs.

\section{References}

[1] Tanmay N \& Arnab G, "Cardiovascular diseases risk factors in Asian Indian Population: A Systematic Review", Journal of Cardiovascular Disease, Pubmed, Vol.4, No.4, (2013), 222-228.

[2] Callaway CW, Donnino MW, Fink EL, Geocadin RG, Golan E, Kern KB \& Zimmerman JL, "Part 8: post-cardiac arrest care: 2015 American Heart Association guidelines update for cardiopulmonary resuscitation and emergency cardiovascular care", Circulation, Vol.132, (2015), pp.465-482.

[3] Goldberger ZD, Chan PS, Berg RA, "For the American Heart Association Get with the guidelines Resuscitation (formerly the National Registry of Cardiopulmonary Resuscitation) Investigators. Duration of resuscitation efforts and survival after in-hospital cardiac arrest: An observation study", Lancet, Vol.380, (2012), pp.1473-81.

[4] Steen S, "Treatment of out-of-hospital cardiac arrest with LUCAS, a new device for automatic mechanical compression and active decompression resuscitation", Resuscitation, Vol.67, No.1, (2005), pp.25-30.

[5] Giraud R, "The LUCAS 2 chest compression device is not always efficient: an echo graphic confirmation", Annals of emergency medicine, Vol.65, No.1, (2015), pp.23-26.

[6] Perkins GD, "Mechanical versus manual chest compression for out-of-hospital cardiac arrest (PARAMEDIC): a pragmatic, cluster randomised controlled trial", Lancet, Vol.385, (2015), pp.947-955.

[7] Physio-Control, Inc., Redmond, WA (US), Front Part for Support Structure for CPR, US Patent No. 8,753,298 A1, (2015).

[8] Weil Institute of Critical Care Medicine, Rancho Mirage, CA (US), "Enhanced Chest Compressor", US 8,790,285 B2, (2007)

[9] Koninklijke Philips Electronics NV, Eindhoven (NL), "Pediatric Patient-Safe CPR Device”, US 2013/0102936 A1, (2011).

[10] Koninklijke Philips Electronics NV, "Eindhoven (NL)," Resuscitation Device", US 2014/0236055 A1, (2012).

[11] Greater Glasgow Health Board, Glasgow, "CPR Assistance Apparatus", WO 2008/025995 A2, (2006). 